\title{
On the Connection between the LSZ and Wightman Quantum Field Theory*
}

\author{
By \\ KLAUS HePP
}

The Institute for Advanced Study, Princeton, New Jersey

\begin{abstract}
The LSZ asymptotic condition and the Yang-Feldman equations are derived in a Wightman quantum field theory on a dense set of scattering states. The Green's distributions are shown to be sufficiently regular around the energy shell to give well-defined reduction formulae for the scattering amplitudes.
\end{abstract}

\section{Introduction}

The analysis of the fundamental notions of relativistic quantum mechanics has been carried out in several axiomatic formalisms [1], which differ significantly in the primary notions involved.

The quantum field theory of Lehmann, Symanzik, and Zimmermann [2] (LSZ) is based on a complete particle interpretation. The $S$-matrix is assumed to be interpolated by a set of local fields satisfying a weak convergence asymptotic condition. Furthermore, one assumes that the fields are such that Green's functions can be defined and are sufficiently regular around the mass shell, in order to give sense to the reduction formulae for the scattering amplitudes. Then the main results of the LSZ theory are analyticity properties of the $S$-matrix elements and the manyparticle structure of Green's functions.

The more general Wightman framework [3] is based on fields which are local and Lorentz covariant operator-valued tempered distributions in a Hilbert space. Under more detailed assumptions on the spectrum of the energy-momentum operator a collision theory has been developed by HaAG [4] and RUelle [5].

In view of the particle interpretation implied by the Haag-Ruelle asymptotic condition, it is natural to investigate the connection between the LSZ and the Wightman framework. The most ambitious program would be to prove that an LSZ theory is a special Wightman theory in which the asymptotic states are complete. More modestly, we intend to show that on a dense set of collision states the LSZ asymptotic condition

* Research supported by the National Science Foundation.

Commun. math. Phys., Vol. 1 
and the Yang-Feldman equations [6] can be proved from the assumptions leading to the Haag-Ruelle scattering theory. The proof is based on the physical idea that collision states describing divergent asymptotic particle configurations can be rapidly approximated by states which are created from the vacuum by polynomials in the fields corresponding to 1-particle excitations.

The asymptotic condition in $x$-space, which is a convergence property at infinity of (regularized) Green's functions in the variables dual to $p^{0}-\left(m^{2}+\mathbf{p}^{2}\right)^{1 / 2}$, gives regularity in $p$-space for the amputated Green's functions around the energy shell $\left\{p^{0}=\left(m^{2}+\mathbf{p}^{2}\right)^{1 / 2}\right\}$. The restriction of these distributions to the energy shell is therefore well-defined, at least for momentum configurations describing divergent particle beams. This will justify the reduction formulae of LSZ for the scattering amplitudes.

\section{Asymptotic Condition}

In this section we shall derive the LSZ asymptotic condition and the Yang-Feldman equations in the framework of the Haag-Ruelle collision theory.

We first restrict ourselves to the theory of one kind of neutral scalar particle with mass $m>0$. We assume that 1-particle states are created from the vacuum $\Omega$ by a neutral scalar Wightman field $A(x)$. This statement is (up to a normalization) equivalent to the following structure of the 2-point function:

$$
\langle A(x) A(y)\rangle_{0}=i \Delta_{+}^{m}(x-y)+i \int_{0}^{\infty} d \varrho(\mu) \Delta_{+}^{\mu}(x-y),
$$

with supp $\varrho \cap\left\{\left|\mu^{2}-m^{2}\right|<c\right\}=\emptyset$ for some $c>0$. Let $G=\left\{p \in R^{4}\right.$ : $\left.p^{0}>0,\left|p^{2}-m^{2}\right|<c\right\}$ and let $\mathscr{S}(G)$ be the space of test functions $f \in \mathscr{S}\left(R^{4}\right)$ with supp $f \subset G$. For any $f \in \mathscr{S}\left(R^{4}\right)$ and all $t$ (with $\omega=\left(\mathbf{p}^{2}+\right.$ $\left.\left.+m^{2}\right)^{1 / 2}\right)$

$$
A(f, t)=(2 \pi)^{-1 / 2} \int d p \tilde{f}(p)^{*} \widetilde{A}(p) \exp \left[-i\left(p^{0}-\omega\right) t\right]
$$

is a bona fide operator in $\mathfrak{g}$. For $f \in \mathscr{S}(G) A^{*}(f, t)$ creates from $\Omega$ a 1-particle state with a wave function $\hat{f}(\mathbf{p})=\tilde{f}(\omega, \mathbf{p}) \in \mathscr{S}\left(R^{3}\right)$ :

$$
\left.A^{*}(f, t) \Omega=\mid \hat{f}\right), \quad A(f, t) \Omega=0 .
$$

Using (2.3) one can construct asymptotic observables. HAAG [4] and RUELLE [5] have shown that for $\tilde{f}_{i} \in \mathscr{S}(G)$

$$
\lim _{t \rightarrow \pm \infty} \prod_{i=1}^{n} A^{(*)}\left(f_{i}, t\right) \Omega
$$

exists in the strong topology in $\mathfrak{G}$. The states $(\mathrm{ex}=\mathrm{in}$, out)

$$
\left.\lim _{t \rightarrow \pm \infty} \prod_{i=1}^{n} A^{*}\left(f_{i}, t\right) \Omega=\mid \hat{f}_{1}, \ldots, \hat{f}_{n}^{\mathrm{ex}}\right)
$$


span Fock spaces of scattering states characterized by initial (final) wave packets $\hat{f}_{1}, \ldots, \hat{f}_{n}$ and outgoing (incoming) waves after (before) collision. If the $\tilde{f}_{i} \in \mathscr{S}\left(R^{4}\right)$ satisfy no restriction on their supports, then one has in weak convergence [7] on $\mathfrak{F}^{\text {ex }}$

$$
\begin{aligned}
\lim _{t \rightarrow \pm \infty}\left(\Phi^{\mathrm{ex}}, \prod_{i=1}^{m} A^{*}\left(f_{i}, t\right) \Omega\right) & =\left(\Phi^{\mathrm{ex}} \mid \hat{f}_{1}, \ldots, \hat{f}_{m}^{\mathrm{ex}}\right) \\
\lim _{t \rightarrow \pm \infty}\left(\Phi^{\mathrm{ex}}, \prod_{i=1}^{m} A\left(f_{i}, t\right) \prod_{j=1}^{n} A^{*}\left(g_{j}, t\right) \Omega\right) & =\left(\Phi^{\mathrm{ex}}, \prod_{i=1}^{m} a_{\mathrm{ex}}(\hat{f}) \mid \hat{g}_{1}, \ldots, \hat{g}_{n}^{\mathrm{ex}}\right)
\end{aligned}
$$

for all $\Phi^{\text {ex }} \in \mathcal{G}^{\text {ex }}$ and $\left\{\tilde{g}_{j}\right\} \subset \mathscr{S}(G)$. Here asymptotic free fields are defined by $A_{\mathrm{ex}}(f)=(2 \pi)^{1 / 2}\left[a_{\mathrm{ex}}^{*}\left(\hat{f}_{1}\right)+a_{\mathrm{ex}}\left(\hat{f}_{2}^{*}\right)\right]$ for $\tilde{f} \in \mathscr{S}\left(R^{4}\right)$ and $\hat{f}_{1}(p)=\tilde{f}(\omega, \mathbf{p})$, $\hat{f}_{2}(\mathbf{p})=\tilde{f}(-\omega,-\mathbf{p})$.

The investigation of asymptotic observables can be carried much further in the LSZ framework, where the $S$-matrix elements are related to Green's functions in momentum space, using a weak convergence asymptotic condition for the field operators. We shall see that a sharpened form of the LSZ asymptotic condition can be proved in the HaagRuelle collision theory. For that purpose we shall first discuss the approach of (2.4) to its limit.

We call $\left\{f_{i}\right\} \subset \mathscr{S}(G)$ non-overlapping, if the supports of the $\left\{f_{i}\right\}$ are pair-wise disjoint in velocity space, i. e., if for all $p_{i}=\left(p_{i}^{0}, \mathbf{p}_{i}\right) \in \operatorname{supp} f_{i}$ one has

$$
\mathbf{p}_{i} \omega_{i}^{-1} \neq \mathbf{p}_{j} \omega_{j}^{-1} \text { for } i \neq j .
$$

A set $\left\{\hat{f}_{i}\right\} \subset \mathscr{S}\left(R^{3}\right)$ is called non-overlapping, if (2.7) holds for all $\mathbf{p}_{i} \in \operatorname{supp} \hat{f}_{i}$. It is a consequence of the short range of the forces in a local quantum field theory with smallest mass $m>0$, that non-overlapping scattering states $\left(\hat{f}_{1}, \ldots, \hat{f}_{n}^{\mathrm{ex}}\right)$ can be approximated rapidly in $t$ by almost localized states $\Pi A^{*}\left(f_{i}, t\right) \Omega$. The proof follows from the

Theorem 2.1: For non-overlapping $\left\{\tilde{f}_{i}\right\} \subset \mathscr{S}(G)$ one has

$$
\left\|\frac{d}{d t} \prod_{i=1}^{n} A^{(*)}\left(f_{i}, t\right) \Omega\right\|<c_{N}(1+|t|)^{-N}
$$

with $c_{N}<\infty$ for all $N$.

Proof: One develops $\left\|\frac{d}{d t} \Pi A^{(*)}\left(f_{i}, t\right) \Omega\right\|^{2}$ into a sum of products of truncated vacuum expectation values [4] (TVEV). Since $A\left(f_{i}, t\right) \Omega$ $=\frac{d}{d t} A^{*}\left(f_{i}, t\right) \Omega=0$, all terms with 1-point functions and pure 2-point functions vanish identically. We shall show that for any $k \geqq 3$ and any $N>0$ :

$$
\left|t^{N}\left\langle\prod_{\nu=1}^{j} A^{(*)}\left(f_{i_{\nu}}, t\right)^{*} \prod_{\nu=j+1}^{k} A^{(*)}\left(f_{i_{\nu}}, t\right)\right\rangle^{T}\right|<c_{N}
$$


is uniformly bounded for all $t$ by some $c_{N}<\infty$. For $k \geqq 3$ one has either $j \geqq 2$ or $k-j \geqq 2$. Suppose $j \geqq 2$ and as the first two factors in (2.9) $A\left(f_{i_{1}}, t\right) A\left(f_{i_{2}}, t\right)$. Using translation invariance $(2.9)$ becomes in momentum space

$$
\left|t^{N} \int \prod_{\nu=1}^{2} d p_{\nu} f_{i_{\nu}}\left(-p_{\nu}\right) e^{i \omega_{\nu} t} \prod_{\nu=3}^{k} d p_{\nu} f_{i_{\nu}}^{(*)}\left( \pm p_{\nu}\right) e^{ \pm i \omega_{\nu} t} \widetilde{W}\left(p_{1}, \ldots, p_{k}\right)^{T}\right|
$$

In $x$-space the TVEV $W\left(x_{1}, \ldots, x_{k}\right)^{T}$ are strongly decreasing for large space-like separation of the arguments $[5,8]$. Therefore $\widetilde{W}^{T}$ is of the form $\delta\left(\sum_{i=1}^{k} p_{i}\right) \hat{W}\left(p_{2}, \ldots, p_{k}\right)^{T}$ with $\hat{W}^{T}$ being $C^{\infty}$ and of polynomial increase in $\mathbf{p}_{2}, \ldots, \mathbf{p}_{k}$, when integrated over $p_{2}^{0}, \ldots, p_{k}^{0}$ with a test function from $\mathscr{S}\left(R^{k-1}\right)$. Thus $(2.10)$ becomes

$$
\begin{gathered}
\left|t^{N} \int \prod_{i=2}^{k} d \mathbf{p}_{i} \chi\left(\mathbf{p}_{2}, \ldots, \mathbf{p}_{k}\right) \exp \left[i \Omega\left(\mathbf{p}_{2}, \ldots, \mathbf{p}_{k}\right) t\right]\right| \\
\chi\left(\mathbf{p}_{2}, \ldots, \mathbf{p}_{k}\right)=\int d p_{1} f_{i_{1}}\left(-p_{1}\right) \prod_{v=2}^{k} d p_{\nu}^{0} f_{i v}^{(*)}\left( \pm p_{v}\right) \widetilde{W}\left(p_{1}, \ldots, p_{k}\right)^{T} \\
\Omega\left(\mathbf{p}_{2}, \ldots, \mathbf{p}_{k}\right)=\left[m^{2}+\left(\sum_{i=2}^{k} \mathbf{p}_{i}\right)^{2}\right]^{1 / 2}+\left(m^{2}+\mathbf{p}_{2}^{2}\right)^{1 / 2} \\
+\sum_{i=3}^{k}\left[ \pm\left(m^{2}+\mathbf{p}_{i}^{2}\right)^{1 / 2}\right]
\end{gathered}
$$

with $\chi \in \mathscr{S}\left(R^{3(k-1)}\right)$. Since $\tilde{f}_{i_{1}}$ and $\tilde{f}_{i_{2}}$ are non-overlapping,

$$
\frac{\partial \Omega}{\partial \mathbf{p}_{2}}=\left[m^{2}+\left(\sum_{i=2}^{k} \mathbf{p}_{i}\right)^{2}\right]^{-1 / 2} \sum_{i=2}^{k} \mathbf{p}_{i}+\omega_{2}^{-1} \mathbf{p}_{2} \neq 0
$$

in the support of $\chi$. By a suitable $\mathcal{O}_{M}$-partition of the unity $\left\{\alpha_{i}\right\}, 1 \leqq$ $\leqq i \leqq 3$, one can arrange that $\partial \Omega / \partial p_{2}^{i} \neq 0$ in $\operatorname{supp} \chi \alpha_{i}$. Then the transformation $\Omega \leftrightarrow p_{2}^{i}$ is regular in supp $\chi \alpha_{i}$. Therefore one has

$\int \Pi \int \mathbf{p}_{v} \chi(\mathbf{p}) \alpha_{i}(\mathbf{p}) \exp [\mathrm{i} \Omega(\mathbf{p}) t] \in \mathscr{S}\left(R^{1}\right)$ for $1 \leqq i \leqq 3$. This proves (2.9) and therefore Theorem 2.1.

It may be remarked, that the majorization extends trivially to arbitrary non-overlapping $\varphi \in \mathscr{S}\left(G^{n}\right)$, which are not necessarily of the type $f_{1} \otimes \cdots \otimes f_{n}$.

The first consequence of theorem 2.1 is that for non-overlapping velocities the Haag-Ruelle limit (2.4) is attained faster than any power in $|t|^{-1}$, independently of the dimension of space in a normal hyperbolic space-time. Hence also in a world of one or two space dimensions, a reasonable collision theory can be developed. 
Let $\tilde{\varphi} \in \mathscr{P}\left(G^{n}\right)$ be non-overlapping and $\mathbb{E}^{n}$ denote the symmetric group on $n$ letters. Define

$$
\begin{aligned}
\Phi(\varphi, t) & =(2 \pi)^{-n / 2} \int d^{4 n} p \tilde{\varphi}(p) \times \\
& \times \exp \left[i \sum_{\nu=1}^{n}\left(p_{\nu}^{0}-\omega_{v}\right) t\right] \widetilde{A}\left(-p_{1}\right) \ldots \widetilde{A}\left(-p_{n}\right) \Omega
\end{aligned}
$$

and $\Phi^{\mathrm{ex}}(\hat{\varphi})$ as its asymptotic limit, which is characterized by the $n$-particle wave function

$$
\hat{\varphi}\left(\mathbf{p}_{1}, \ldots, \mathbf{p}_{n}\right)=(n !)^{-1 / 2} \sum_{Q \in \mathbb{E}^{n}} \varphi\left(\omega_{Q(1)}, \mathbf{p}_{Q(1)}, \ldots, \omega_{Q(n)}, \mathbf{p}_{Q(n)}\right) .
$$

Let $B$ be an arbitrary polynomial in the smeared-out field. By the Schwarz inequality one has

$$
\left\|B \frac{d}{d t} \Phi(\varphi, t)\right\|^{2} \leqq\left\|\frac{d}{d t} \Phi(\varphi, t)\right\|\left\|B^{*} B \frac{d}{d t} \Phi(\varphi, t)\right\| .
$$

The second factor on the right-hand side of (2.17) increases with a fixed power of $|t|$ due to the temperedness of the Wightman distributions, while the first factor decreases stronger than any power of $|t|$ for nonoverlapping $\tilde{\varphi} \in \mathscr{S}\left(G^{n}\right)$. Therefore $B \Phi(\varphi, t)$ again converges strongly for $t \rightarrow \pm \infty$. Let $\mathfrak{P}$ be the algebra of all polynomials in the smearedout fields. By assumption [3]P $\Omega$ is dense in $\mathfrak{G}$ and belongs to the domain of $B^{*}$. Therefore the closure $\bar{B}=B^{* *}$ of $B$ exists. By definition of $\bar{B}$ one has

$$
\lim _{t \rightarrow \pm \infty} B \Phi(\varphi, t)=\bar{B} \Phi^{\mathrm{ex}}(\hat{\varphi})
$$

and (again using the definition of the closure) for $B_{1}, B_{2} \in \mathfrak{P}$ :

$$
B_{1} B_{2} \Phi(\varphi, t) \rightarrow \overline{B_{1} B_{2}} \Phi^{\mathrm{ex}}(\hat{\varphi})=\bar{B}_{1}\left(\overline{B_{2}} \Phi^{\mathrm{ex}}(\hat{\varphi})\right) .
$$

Let $D_{0}^{\mathrm{ex}}$ be the linear space of all non-overlapping $\Phi^{\mathrm{ex}}(\hat{\varphi})$ with $\tilde{\varphi} \in \mathscr{S}\left(G^{n}\right)$, $n=0,1,2, \ldots D_{0}^{\mathrm{ex}}$ is dense in $\mathfrak{G}^{\mathrm{ex}}$, since the functions (2.16) are dense in the $L^{2}$-space of all totally symmetric $\varphi\left(\mathbf{p}_{1}, \ldots, \mathbf{p}_{n}\right)$ with $\int \prod d p_{i} \delta_{+}\left(p_{i}\right)$ $\times\left|\varphi\left(\mathbf{p}_{1}, \ldots, \mathbf{p}_{n}\right)\right|^{2}<\infty$.

The spaces $D_{0}^{\mathrm{ex}}$ share many important properties with $\mathfrak{P} \Omega$, where the field operators are originally defined. $D_{0}^{\text {ex }}$ lies in the intersection of the domains of all $\bar{B}, B \in \mathfrak{P}$, and all $a_{\mathrm{ex}}^{(*)}(\hat{f})$ with square integrable testing functions.

Due to (2.19) the $\bar{B}, B \in \mathfrak{P}$, can be freely multiplied on $D_{0}^{\mathrm{ex}} \cdot\left[\bar{B}_{1}, \bar{B}_{2}\right]=0$ holds on $D_{0}^{\text {ex }}$ if the test functions corresponding to $B_{1}, B_{2} \in \mathfrak{P}$ have spacelike supports. The unitarity of the representation $U(a, \Lambda)$ of $i L_{+}^{\uparrow}$ entails on $D_{0}^{\text {ex }}$ :

$$
U(a, \Lambda) \bar{B} U(a, \Lambda)^{-1}=\overline{U(a, \Lambda) B U(a, \Lambda)^{-1}} .
$$


Finally, let $\psi_{v} \in \mathscr{S}\left(R^{4 n}\right)$ and $\Phi^{\text {out }}(\hat{\varphi}) \in D_{0}^{\text {out }}$. Then $B\left(\psi_{v}\right) \equiv \int d x_{1} \ldots$ $d x_{n} \psi_{v}\left(x_{1}, \ldots, x_{n}\right) A\left(x_{1}\right) \ldots A\left(x_{n}\right)$ satisfies

$$
\left\|\overline{B\left(\psi_{v}\right)} \Phi^{\text {out }}(\hat{\varphi})\right\| \leqq\left\|B\left(\psi_{v}\right) \Phi(\varphi, t)\right\|+\int_{t}^{\infty} d s\left\|\frac{d}{d s} B\left(\psi_{v}\right) \Phi(\varphi, s)\right\| .
$$

The squared integrand can be majorized using (2.17) by a finite sum

$$
\sum_{\alpha, \beta, \gamma, \delta} \sup _{x, y}|x|^{\alpha}\left|D_{x}^{\beta} \psi_{\nu}(x)\right||y|^{\mid}\left|D_{y}^{\delta} \psi_{\nu}(y)\right| C_{\alpha \beta \gamma \delta}^{N}(1+|s|)^{-N}
$$

with $C_{\alpha \beta \gamma \delta}^{N}<\infty$ for all $N \geqq 0$. Therefore $\psi_{\nu} \rightarrow 0$ in $\mathscr{S}\left(R^{4}\right)$ entails $\left\|\overline{B\left(\psi_{v}\right)} \Phi^{\mathrm{ex}}(\hat{\varphi})\right\| \rightarrow 0$.

These properties of $D_{0}^{\text {ex }}$ motivate the "abus de langage" $B \Phi^{\text {ex }}$ for $\bar{B}$ operating on $D_{0}^{\mathrm{ex}}$.

Now the ground is laid for a proof of the LSZ asymptotic condition given independently by HAAG and RoBINSON [9] using similar methods:

Theorem 2.2: Let $\Phi^{\mathrm{ex}} \in D_{0}^{\mathrm{ex}}$ and $\left\{\tilde{f}_{i}\right\} \subset \mathscr{S}(G)$, then

$$
\lim _{t \rightarrow \pm \infty} \prod_{i=1}^{n} A^{(*)}\left(f_{i}, t\right) \Phi^{\mathrm{ex}}=\prod_{i=1}^{n} a_{\mathrm{ex}}^{(*)}\left(\hat{f}_{i}\right) \Phi^{\mathrm{ex}} .
$$

For $\Phi^{\mathrm{ex}} \in D_{0}^{\mathrm{ex}}, \Psi^{\mathrm{ex}} \in \mathcal{F}^{\mathrm{ex}},\left\{\tilde{f}_{i}\right\} \subset \mathscr{S}(G),\left\{\tilde{g}_{i}\right\} \subset \mathscr{S}\left(R^{4}\right)$ one has

$$
\begin{gathered}
\lim _{t \rightarrow \pm \infty}\left(\Psi^{\mathrm{ex}}, \prod_{i=1}^{m} A^{*}\left(g_{i}, t\right) \Phi^{\mathrm{ex}}\right)=\left(\Psi^{\mathrm{ex}}, \prod_{i=1}^{m} a_{\mathrm{ex}}^{*}\left(\hat{g}_{i}\right) \Phi^{\mathrm{ex}}\right) \\
\lim _{t \rightarrow \pm \infty}\left(\Psi^{\mathrm{ex}}, \prod_{i=1}^{m} A\left(g_{i}, t\right) \prod_{j=1}^{n} A^{*}\left(f_{j}, t\right) \Phi^{\mathrm{ex}}\right) \\
=\left(\Psi^{\mathrm{ex}}, \prod_{i=1}^{m} a_{\mathrm{ex}}\left(\hat{g}_{i}\right) \prod_{j=1}^{n} a_{\mathrm{ex}}^{*}\left(\hat{f}_{j}\right) \Phi^{\mathrm{ex}}\right) .
\end{gathered}
$$

Proof: We first remark that for $\Phi^{\text {ex }} \in D_{0}^{\text {ex }}(2.23)$ and (2.24) are well-defined. We approximate $\prod_{i=1}^{n} A^{(*)}\left(f_{i}, t\right) \Phi^{\mathrm{ex}}(\hat{\varphi})$ by $\prod_{i=1}^{n} A^{(*)}\left(f_{i}, t\right)$ $\Phi(\varphi, t)$, which is possible due to Theorem 2.1:

$$
\begin{aligned}
& \left\|\prod_{i=1}^{n} A^{(*)}\left(f_{i}, t\right) \Phi^{\mathrm{out}}(\hat{\varphi})-\prod_{i=1}^{n} A^{(*)}\left(f_{i}, t\right) \Phi(\varphi, t)\right\| \leqq \\
\leqq & \int_{i}^{\infty} d s\left\|\prod_{i=1}^{n} A^{(*)}\left(f_{i}, t\right) \frac{d}{d s} \Phi(\varphi, s)\right\|<C_{N}(1+|t|)^{-N},
\end{aligned}
$$

since the integrand can be majorized as in (2.17) by $d_{K}(1+|s|)^{-K}(1+|t|)^{L}$, with $L$ fixed and $d_{K}<\infty$ for all $K$. Then (2.23) and (2.24) follow from the Haag-Ruelle theorem (2.4) and (2.6). 
The generalization of theorems 2.1 and 2.2 to arbitrary spins and bound-states is not difficult. If a general $i S L(2, C)$-covariant $(2 r+1) \times$ $(2 t+1)$ comporent field [3] $A_{\varrho \dot{\tau}}(x)$ with

$$
U(a, \Lambda) A_{\varrho \dot{i}}(x) U(a, \Lambda)^{-1}=D_{\varrho \varrho^{\prime}}^{r}\left(\Lambda^{-1}\right) D_{\tilde{i} \dot{t}^{\prime}}^{t}\left(\Lambda^{-1}\right)^{*} A_{\varrho^{\prime} \dot{i}^{\prime}}(\Lambda x+a)
$$

creates from $\Omega$ a 1-particle state of mass $m>0$ and spin $s(|r+t| \geqq s \geqq$ $\geqq|r-t|)$, then there exists [5, 10] a local $(2 s+1)$ component field $A_{\sigma}(x)$, whose 2-point function $\left\langle A_{\sigma}(x) A_{i}^{*}(y)\right\rangle_{0}$ coincides (up to normalization) with the 2-point function of the corresponding free field in a neighborhood $G$ of the energy shell $\left(\delta_{+}(p) \equiv \theta\left(p^{0}\right) \delta\left(p^{2}-m^{2}\right)\right)$ :

$$
\begin{aligned}
\left\langle A_{\sigma}(x) A_{i}^{*}(y)\right\rangle_{0} & =(2 \pi)^{-3} \int d p \delta_{+}(p) D_{\sigma \dot{\tau}}^{s}\left[\frac{1}{m}\left(p^{0}+\mathbf{p} \boldsymbol{\sigma}\right)\right] e^{-i p(x-y))}+ \\
& +(\text { continuum contribution) }
\end{aligned}
$$

Then the Haag-Ruelle and the LSZ asymptotic condition can be proved using

$$
A(f, t)=(2 \pi)^{-1 / 2} \sum_{\sigma=1}^{2 s+1} \int d p \tilde{f}_{\sigma}(p)^{*} \widetilde{A}_{\sigma}(p) \exp \left[-i\left(p^{0}-\omega\right) t\right]
$$

with $f_{\sigma} \in \mathscr{S}(G)$ or $\in \mathscr{S}\left(R^{4}\right)$ for strong or weak convergence. In the case of a bound state a polynomial $B$ in the smeared-out fields is assumed to create from $\Omega$ a 1-particle state corresponding to $[m, s]$ (i. e., $B^{*} \Omega \in \mathfrak{S}_{[m, s]}$ and $B \Omega=0$ ). Then Theorems 2.1 and 2.2 follow using the limiting procedure $[4,5]$.

$$
B(f, t)=i \int_{x^{0}=t} d \mathbf{x} f^{*}(x) \breve{\partial}_{0}\left[U(x, 1) B U^{-1}(x, 1)\right]
$$

with $f(x)=(2 \pi)^{-3 / 2} \int \delta_{+}(p) \hat{f}(p) e^{-i p x} d p, \hat{f} \in \mathscr{S}\left(R^{3}\right)$ and $\overrightarrow{\partial_{0}}=\overrightarrow{\partial \mid \partial} x_{0}-$ $-\overleftarrow{\partial \partial x_{0}}$. Here weak convergence holds again even if $E_{[m, s]} B^{*} \Omega \neq B^{*} \Omega$, with $E_{[m, s]}$ projector on $\mathfrak{G}_{[m, s]}$, if $m_{i} \neq m_{j}$ for different $\left[m_{i}, s_{i}\right]$.

In conclusion we discuss another formulation of the asymptotic condition which is currently believed to be equivalent to the axiom of LSZ: the definition of asymptotic fields by the Yang-Feldman equations [6].

For any $f \in \mathscr{S}\left(R^{4}\right)$ there exist $f_{i} \in \mathscr{S}\left(R^{4}\right), 1 \leqq i \leqq 3$, with $f(p)$ $=\sum f_{i}(p)$ and

$$
\begin{aligned}
& \operatorname{supp} \tilde{f}_{1} \subset\left\{\left|p^{2}-m^{2}\right|<c, p^{0}>0\right\} \\
& \text { supp } f_{2} \subset\left\{\left|p^{2}-m^{2}\right|<c, p^{0}<0\right\} \\
& \text { supp } f_{3} \cap\left\{\left|p^{2}-m^{2}\right|<\frac{c}{2}\right\}=\phi .
\end{aligned}
$$

The $C^{\infty}$-function $F_{1}(s)=\frac{d}{d s}(2 \pi)^{1 / 2} A^{*}\left(f_{1}, s\right) \Psi^{\text {in }}$ satisfies for $\Psi^{\text {in }} \in D_{0}^{\text {in }}$ the majorization $\left\|F_{1}(s)\right\|<C_{1}(1+|s|)^{-3 / 2}$ in $-\infty<s \leqq 0$. Therefore $\int_{-\infty}^{0} e^{\varepsilon s} \times$ 
$\times F_{1}(s) d s$ is uniformly convergent for $0 \leqq \varepsilon \leqq \varepsilon_{0}, \varepsilon_{0}>0$, and one obtains :

$$
\begin{aligned}
\int_{-\infty}^{0} F_{1}(s) d s & =\lim _{\varepsilon \downarrow 0} \int_{-\infty}^{0} e^{\varepsilon s} F_{1}(s) d s \\
& =\lim _{\varepsilon \downarrow 0} \int_{-\infty}^{0} d s i \int d p \tilde{f}_{1}(p) \times \\
& \times \exp \left[i s\left(p^{0}-\omega-i \varepsilon\right)\right]\left(p^{0}-\omega\right) \widetilde{A}(-p) \Psi^{\text {in }} \\
& =-\lim _{\varepsilon \downarrow 0} \int d p \tilde{f}_{1}(p)\left[\left(p^{0}+\omega\right)\left(p^{0}-\omega-i \varepsilon\right)\right]^{-1} \tilde{\jmath}(-p) \Psi^{\text {in }} \\
& =A\left(f_{1}\right) \Psi^{\text {in }}-A_{\text {in }}\left(f_{1}\right) \Psi^{\text {in }} .
\end{aligned}
$$

Here one uses Theorem 2.1. Furthermore one uses $p^{0}+\omega>c>0$ in $\operatorname{supp} f_{1}, \tilde{j}(p)=\left(-p^{2}+m^{2}\right) \widetilde{A}(p)$, and for $\varepsilon>0$ in the topology of $\mathscr{S}\left(R^{4}\right)$ :

$$
\lim _{s \rightarrow-\infty} \frac{\tilde{f}_{1}(p) \exp \left[i s\left(p^{0}-\omega-i \varepsilon\right)\right]}{\left(p^{0}+\omega\right)\left(p^{0}-\omega-i \varepsilon\right)}=0 .
$$

Similarly one shows that

$$
\begin{aligned}
& A\left(f_{2}\right) \Psi^{\text {in }}-A_{\text {in }}\left(f_{2}\right) \Psi^{\text {in }} \\
& \quad=-\lim _{\varepsilon \downarrow 0} \int d p \tilde{f}_{2}(p)\left[\left(p^{0}+\omega-i \varepsilon\right)\left(p^{0}-\omega\right)\right]^{-1} \tilde{\jmath}(-p) \Psi^{\text {in }} .
\end{aligned}
$$

Since the mass shell lies outside of the support of $f_{3}$, one finally has

$$
A_{\text {in }}\left(f_{3}\right)=0 \text { and } A\left(f_{3}\right) \Psi^{\text {in }}=-\int d p \tilde{f}_{3}(p)\left[p^{2}-m^{2}\right]^{-1} \tilde{\jmath}(-p) \Psi^{\text {in }} .
$$

This gives in $x$-space the

Theorem 2.3: For $\Psi^{\mathrm{ex}} \in D_{0}^{\mathrm{ex}}$ the Yang-Feldman equations hold as distribution identity in $\mathscr{S}^{\prime}\left(R^{4}\right)$ :

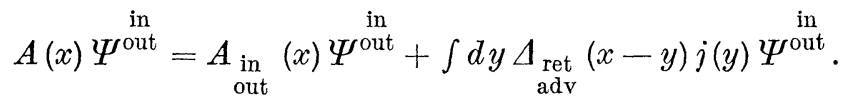

\section{Reduction Formulae}

The well-known reduction formulae [2] for the scattering amplitudes are usually derived with help of the LSZ asymptotic condition. In the Wightman framework they are not trivially obtainable, because they require a knowledge of the singularity structure of (not well-defined) Green's functions around the energy shell. But, since the LSZ asymptotic condition can be proved in a strong form on non-overlapping states as a consequence of the more fundamental Theorem 2.1, we expect to gain a deeper insight into the structure of the LSZ formalism by deriving somehow similar reduction formulae in the Wightman framework.

We avoid all existence questions of Green's functions [11] in terms of Wightman distributions by using regularized characteristic functions. 
The connection with the scattering amplitudes will turn out to be independent of the regularization and will extend immediately to "sharp" Green's functions, whenever the latter can be defined. Throughout this chapter we assume (2.1) with a mass eigenvalue $m^{2}$ of $P^{2}$ outside the continuum.

Let $\chi \in \mathscr{D}\left(R^{n-1}\right)$ be arbitrary except that it satisfies for all $P \in \mathbb{S}^{n}$ :

$$
\begin{gathered}
\chi\left(s_{P(1)}-s_{P(2)}, \ldots, s_{P(n-1)}-s_{P(n)}\right)=\chi\left(s_{1}-s_{2}, \ldots, s_{n-1}-s_{n}\right), \\
\int d t_{1} \ldots d t_{n-1} \chi\left(t_{1}, \ldots, t_{n-1}\right)=1 .
\end{gathered}
$$

Then "smooth" time-ordered, advanced and retarded products are defined by

$$
\begin{gathered}
T_{\chi}\left(x_{1}, \ldots, x_{n}\right)=\int d\left(s_{1}-s_{2}\right) \ldots d\left(s_{n-1}-s_{n}\right) \times \\
\times \chi\left(s_{1}-s_{2}, \ldots, s_{n-1}-s_{n}\right) \sum_{P \in \mathbb{S}^{n}} \theta\left(x_{P(1)}^{0}-s_{P(1)}-x_{P(2)}^{0}+s_{P(2)}\right), \ldots, \\
\theta\left(x_{P(n-1)}^{0}-s_{P(n-1)}-x_{P(n)}^{0}+s_{P(n)}\right) A\left(x_{P(1)}\right) \ldots A\left(x_{P(n)}\right) \\
A_{\chi}\left(x_{1} ; x_{2}, \ldots, x_{n}\right)=i^{n-1} \int d\left(s_{1}-s_{2}\right) \ldots d\left(s_{n-1}-s_{n}\right) \times \\
\times \chi\left(s_{1}-s_{2}, \ldots, s_{n-1}-s_{n}\right) \times \\
\times \sum_{P \in \mathbb{E}^{n-1}} \theta\left(x_{P(n)}^{0}-s_{P(n)}-x_{P(n-1)}^{0}+s_{P(n-1)}\right) \ldots \\
\theta\left(x_{P(2)}^{0}-s_{P(2)}-x_{1}^{0}+s_{1}\right)\left[\left[A\left(x_{1}\right) A\left(x_{P(2)}\right)\right], \ldots, A\left(x_{P(n)}\right)\right]
\end{gathered}
$$

and similarly $R_{\chi}\left(x_{1} ; x_{2}, \ldots, x_{n}\right)$.

The following simple reduction formula [2] is well known from LSZ. Let $T_{x}\left(x_{1}, \ldots, x_{n}\right)$ be any smooth, time-ordered product. Then for sufficiently large $R>0$ one has

$$
T_{\chi}\left(x_{1}, \ldots, x_{n}\right)=\left\{\begin{array}{l}
T_{\chi}\left(x_{2}, \ldots, x_{n}\right) A\left(x_{1}\right), \text { for } x_{i}-x_{1}>R(2 \leqq i \leqq n) \\
A\left(x_{1}\right) T_{z}\left(x_{2}, \ldots, x_{n}\right), \text { for } x_{1}-x_{i}>R(2 \leqq i \leqq n)
\end{array}\right.
$$

where $T_{\chi}\left(x_{2}, \ldots, x_{n}\right)$ is defined as in (3.2) by restricting $\sum_{P}$ to those $P \in \mathfrak{S}^{n}$ with $P(1)=1$ and by replacing $\theta\left(x_{1}^{0}-s_{1}-x_{P(2)}^{0}+s_{P(2)}\right)$ by 1 . Then one can derive for $\Phi^{\text {out }} \in D_{0}^{\text {out }}, \Psi^{\text {in }} \in D_{0}^{\text {in }}$ the following distribution identity in $\mathscr{S}^{\prime}\left(G \times R^{4(n-1)}\right)$ :

$\left(a_{\text {out }}\left(\mathbf{p}_{1}\right) \Phi^{\text {out }}, \widetilde{T}_{\chi}\left(-p_{2}, \ldots,-p_{n}\right) \Psi^{\text {in }}\right)-\left(\Phi^{\text {out }}, \widetilde{T}_{\chi}\left(-p_{2}, \ldots,-p_{n}\right) \times\right.$ $\left.a_{\text {in }}^{*}\left(\mathbf{p}_{1}\right) \Psi^{\text {in }}\right)=\left.i(2 \pi)^{1 / 2}\left[\left(p_{1}^{2}-m^{2}\right)\left(\Phi^{\text {out }}, \widetilde{T}_{\chi}\left(-p_{1}, \ldots,-p_{n}\right) \Psi^{\text {in }}\right)\right]\right|_{p_{1}^{0}=\omega_{1}}$.

Here the restriction of the tempered distribution $\left(p_{1}^{2}-m^{2}\right)\left(\Phi^{\text {out }}, \widetilde{T}_{x}\left(-p_{1}\right.\right.$, $\left.\ldots,-p_{n}\right) \Psi^{\text {in }}$ ) to the energy shell will turn out to be well-defined as a continuous function in the critical variable $p_{1}^{0}-\omega_{1}$.

For, take $\tilde{\psi} \in \mathscr{S}\left(G \times R^{4(n-1)}\right)$. Since the Fourier transform of $\tilde{\psi} \exp \left[i\left(p_{1}^{0}-\omega_{1}\right) t\right]$ decreases in $x$-space strongly in $x_{1}^{0}-t$, one has for 
$t \rightarrow+\infty:$

$\int \Pi d p_{i} \tilde{\psi}(p) \exp \left[i\left(p_{1}^{0}-\omega_{1}\right) t\right]\left(\Phi^{\text {out }}, \tilde{T}_{\chi}\left(-p_{1}, \ldots,-p_{n}\right) \Psi^{\text {in }}\right)$

$\rightarrow \int \Pi d p_{i} \tilde{\psi}(p) \exp \left[i\left(p_{1}^{0}-\omega_{1}\right) t\right]\left(\widetilde{A}\left(p_{1}\right) \Phi^{\text {out }}, \widetilde{T}_{\chi}\left(-p_{2}, \ldots,-p_{n}\right) \Psi^{\text {in }}\right)(3.5)$

$\rightarrow(2 \pi)^{1 / 2} \int \Pi d p_{i} \delta_{+}\left(p_{1}\right) \tilde{\psi}(p)\left(a_{\text {out }}\left(\mathbf{p}_{1}\right) \Phi^{\text {out }}, \tilde{T}_{\chi}\left(-p_{2}, \ldots,-p_{n}\right) \Psi^{\text {in }}\right)$

as $|t|^{-1 / 2}$, due to the LSZ asymptotic condition. For $t \rightarrow-\infty$ the lefthand side of (3.5) converges to

$$
(2 \pi)^{1 / 2} \int \Pi d p_{i} \delta_{+}\left(p_{1}\right) \tilde{\psi}(p)\left(\Phi^{\mathrm{out}}, \tilde{T}_{\chi}\left(-p_{2}, \ldots,-p_{n}\right) a_{\mathrm{in}}^{*}\left(\mathbf{p}_{1}\right) \Psi^{\mathrm{in}}\right) .
$$

Therefore

$$
\begin{gathered}
\int \prod_{i=1}^{n} d p_{i} \delta_{+}\left(p_{1}\right) \tilde{\psi}(p)\left\{\left(a_{\text {out }}\left(\mathbf{p}_{1}\right) \Phi^{\text {out }}, \tilde{T}_{\chi}\left(-p_{2}, \ldots,-p_{n}\right) \Psi^{\text {in }}\right)-\right. \\
\left.-\left(\Phi^{\text {out }}, \tilde{T}_{\chi}\left(-p_{2}, \ldots,-p_{n}\right) a_{\text {in }}^{*}\left(\mathbf{p}_{1}\right) \Psi^{\text {in }}\right)\right\} \\
=i(2 \pi)^{-1 / 2} \int_{-\infty}^{+\infty} d t \int d^{4 n} p \tilde{\psi}(p)\left(p_{1}^{0}+\omega_{1}\right)^{-1} \exp \left[i\left(p_{1}^{0}-\omega_{1}\right) t\right]\left(\Phi^{\text {out }},\right. \\
\left.\times\left(p_{1}^{2}-m^{2}\right) \tilde{T}_{\chi}\left(-p_{2}, \ldots,-p_{n}\right) \Psi^{\text {in }}\right) .
\end{gathered}
$$

The $t$-integrand in (3.6) can be majorized by $C(1+|t|)^{-3 / 2}$. Therefore [12] its Fourier transform is continuous in the variable $p_{1}^{0}-\omega_{1}$, after integration over $\mathbf{p}_{1}, p_{2}, \ldots, p_{n}$, and the well-defined restriction to the energy shell gives (3.4).

In the same sense [with $f(x)$ as in (2.28)], the following reduction formulae are well-defined:

$$
\begin{gathered}
a_{\text {out }}(\hat{f}) T_{\chi}\left(x_{2}, \ldots, x_{n}\right)-T_{\chi}\left(x_{2}, \ldots, x_{n}\right) a_{\text {in }}(\hat{f}) \\
=-i \int d x_{1} f^{*}\left(x_{1}\right) K_{1} T_{\chi}\left(x_{1}, \ldots, x_{n}\right), \\
{\left[R_{\chi}\left(x_{0} ; x_{1}, \ldots, x_{n-1}\right), a_{\text {in }}^{*}(\hat{f})\right]=\int d x_{n} f\left(x_{n}\right) K_{n} R_{\chi}\left(x_{0} ; x_{1}, \ldots, x_{n}\right),} \\
{\left[A_{\chi}\left(x_{0} ; x_{1}, \ldots, x_{n-1}\right), a_{\text {out }}^{*}(\hat{f})\right]=\int d x_{n} f\left(x_{n}\right) K_{n} A_{\chi}\left(x_{0} ; x_{1}, \ldots, x_{n}\right) .}
\end{gathered}
$$

(3.7) holds between $\Phi^{\text {out }} \in D_{0}^{\text {out }}$ and $\Psi^{\text {in }} \in D_{0}^{\text {in }},(3.8)$ and (3.9) between states from $D_{0}^{\text {in }}$ and $D_{0}^{\text {out }}$, respectively. Obviously these formulae remain valid, if "sharp" Green's functions can be defined.

The simple support argument leading to formula (3.4) allows a generalization, which first turned up in Zimmermann's study of the 1-particle singularities of the Green's functions [13]. Let $\tilde{\varphi} \in \mathscr{S}\left(R^{4 n}\right)$ have support in $q=p_{1}+\cdots+p_{m}$ in $G$ and consider

$$
F(t)=\int \prod_{i} d p_{i} \tilde{\varphi}(p) \exp \left[i\left(q^{0}-\omega_{q}\right) t\right]\left\langle\tilde{T}_{\chi}\left(-p_{1}, \ldots,-p_{n}\right)\right\rangle_{0} .
$$

Since the Fourier transform of $\tilde{\varphi} \exp \left[i\left(q^{0}-\omega_{q}\right) t\right]$ is concentrated in $x$-space around $x_{i}^{0} \approx t(1 \leqq i \leqq m), x_{j}^{0} \approx 0(m+1 \leqq j \leqq n)$, one can for $t \rightarrow+\infty$ replace $\left\langle\widetilde{T}_{\chi}\left(-p_{1}, \ldots,-p_{n}\right)\right\rangle_{0}$ by $\left\langle\tilde{T}_{\chi}\left(-p_{1}, \ldots,-p_{m}\right) \times\right.$ $\left.\times \tilde{T}_{\chi}\left(-p_{m+1} \ldots-p_{n}\right)\right\rangle_{0}$ up to terms $0\left(|t|^{-N}\right)$. The latter distribution has 
supportin $q \in \bar{V}_{-}$and gives no contribution to (3.10). Similarly, one has for $t \rightarrow-\infty$ :

$$
\begin{aligned}
\mid F(t) & -\int \Pi d p_{i} \tilde{\varphi}\left(p_{1}, \ldots, p_{n}\right) \times \\
& \times\left\langle\tilde{T}_{\chi}\left(-p_{m+1}, \ldots,-p_{n}\right) \tilde{T}_{\chi}\left(-p_{1}, \ldots,-p_{m}\right)\right\rangle \quad \mid<C_{N}(1+|t|)^{-N} .
\end{aligned}
$$

The property $\frac{d}{d t} F(t) \in \mathscr{S}\left(R^{1}\right)$ is equivalent to the statement that $\left(q^{2}-m^{2}\right)\left\langle\tilde{T}_{\chi}\left(-p, \ldots,-p_{n}\right)\right\rangle_{0}$ is $C^{\infty}$ in $q^{0}-\omega_{q}$ around the origin, when integrated over the remaining variables. After an allowed interchange of integrations one obtains

$$
\begin{gathered}
\lim _{t \rightarrow-\infty} F(t)=-\int_{-\infty}^{+\infty} d t \frac{d}{d t} F(t) \\
=-2 \pi i \int \Pi d p_{i} \tilde{\varphi}(p) \delta_{+}(q)\left[\left(q^{2}-m^{2}\right)\left\langle\widetilde{T}_{\chi}\left(-p_{1}, \ldots,-p_{n}\right)\right\rangle_{0}\right] \quad(3.11) \\
=\int \Pi d p_{i} \tilde{\varphi}\left(p_{1}, \ldots, p_{n}\right)\left\langle\tilde{T}_{\chi}\left(-p_{m+1}, \ldots,-p_{n}\right) \tilde{T}_{\chi}\left(-p_{1}, \ldots,-p_{m}\right)\right\rangle_{0} .
\end{gathered}
$$

This is Zimmermann's formula [13] for the 1-particle singularity of $\left\langle\widetilde{T}_{\chi}\left(-p_{1}, \ldots,-p_{n}\right)\right\rangle_{0}$ in $q=\sum_{i=1}^{n} p_{i}$. When "sharp" time-ordered distributions can be defined, one can obtain from (3.11) and (3.4) the "residuum": $2 \pi \int d k d^{4 n} p \tilde{\varphi}(p) \delta_{+}(k)\left[\left(k^{2}-m^{2}\right)\left\langle\tilde{T}\left(k,-p_{1}, \ldots,-p_{m}\right)\right\rangle_{0}\left(k^{2}-m^{2}\right) \times\right.$

$$
\left.\times\left\langle\tilde{T}\left(-p_{m+1}, \ldots,-p_{n},-k\right)\right\rangle_{0}\right] \text {. }
$$

Computing $F(0)=-\int_{0}^{\infty} d t \frac{d}{d t} F(t)$ as in theorem 2.3 one obtains in the topology of $\mathscr{S}^{\prime}\left(R^{4 n}\right)$ :

$$
\left\langle\tilde{T}_{\chi}\left(-p_{1}, \ldots,-p_{n}\right)\right\rangle_{0}=\lim _{\varepsilon \downarrow 0} \frac{\left[\left(q^{2}-m^{2}\right)\left\langle\tilde{T}_{\chi}\left(-p_{1}, \ldots,-p_{n}\right)\right\rangle_{0}\right]}{q^{2}-m^{2}+i \varepsilon} .
$$

$F_{i r r}(t) \equiv F(t)-F(-\infty) \theta(-t)$ is $C^{\infty}$ in $t$ except for a discontinuity at $t=0$ and satisfies $\left|F_{i r r}(t)\right|<C_{N}(1+|t|)^{-N}$ with $C_{N}<\infty$ for all $N$. Therefore

$$
\left\langle\tilde{T}_{\chi}\left(-p_{1}, \ldots,-p_{n}\right)\right\rangle_{0}-\left.\lim _{\varepsilon \downarrow 0} \frac{\left[\left(q^{2}-m^{2}\right)\left\langle\tilde{T}_{\chi}\left(-p_{1}, \ldots,-p_{n}\right)\right\rangle\right]}{q^{2}-m^{2}+i \varepsilon}\right|_{q^{0}=\omega_{q}}
$$

is regular in $q^{0}-\omega_{q}$ around the origin, when integrated over the remaining variables.

Let us remark that the formulae (3.11), (3.12) and (3.13) were derived only from mass spectrum conditions, without the asymptotic condition (based on locality). This possibility was first realized by RUELLE [14] and STREATER [15] by studying the absorptive part of the generalized retarded functions. A similar argument as above fails to exhibit the 1-particle structure of the retarded distributions given first by SYMANzIK [16]. 
Locality and mass spectrum conditions in the form of the LSZ asymptotic condition are an important tool to determine the singularity structure of Green's functions simultaneously in several variables around the energy shell. Our main result is expressed in the following:

Theorem 3.1: The distribution

$$
\prod_{i=k+1}^{l}\left(p_{i}^{2}-m^{2}\right)\left\langle\mathbf{p}_{1}, \ldots, \mathbf{p}_{k}^{\text {out }}\left|\widetilde{T}_{\chi}\left( \pm p_{k+1}, \ldots, \pm p_{l}\right)\right| \mathbf{p}_{l+1}, \ldots, \mathbf{p}_{m}^{\text {in }}\right\rangle
$$

is $C^{\infty}$ in the variables $p_{k+1}^{0}-\omega_{k+1}, \ldots, p_{l}^{0}-\omega_{l}$ around the origin, when integrated over $\mathbf{p}_{1}, \ldots, \mathbf{p}_{m}$ with testing functions $\psi \in \mathscr{S}\left(R^{3 m}\right)$ having pairwise disjoint supports in the variables $\mathbf{p}_{i} \omega_{i}^{-1}$.

Proof: Take non-overlapping $\left\{f_{i}\right\} \subset \mathscr{S}(G)$ and define $f_{i}(x, t) \equiv$ $\equiv \mathscr{F}\left\{\tilde{f}_{i} \exp \left[i\left(p^{0}-\omega\right) t\right]\right\}(x)$ and $\dot{f}_{i}(x, t) \equiv \partial / \partial t f_{i}(x, t)$. Then we claim that

$$
\int \prod_{i=k+1}^{l} d x_{i} \dot{f}_{i}^{(*)}\left(x_{i}, t_{i}\right)\left\langle\hat{f}_{1}, \ldots, \hat{f}_{k}^{\text {out }}\left|T_{\chi}\left(x_{k+1}, \ldots, x_{l}\right)\right| \hat{f}_{l+1}, \ldots, \hat{f}_{m}^{\text {in }}\right\rangle
$$

lies in $\mathscr{S}\left(R^{l-k}\right)$ in the variables $t_{k+1}, \ldots, t_{l}$. Since all the $t$-derivatives of the $C^{\infty}$ function (3.15) are again of the type (3.15), it is sufficient to show that for all $N_{k+1}, \ldots, N_{l} \geqq 0$

$$
\begin{aligned}
\sup _{t_{k+1}, \ldots, t_{l}} \mid \int \prod_{i=k+1}^{l} d x_{i} t_{i}^{N_{i}} \dot{f}_{i}^{(*)}\left(x_{i}, t_{i}\right)\left\langle\hat{f}_{i}, \ldots, \hat{f}_{k}^{\text {out }}\right| \times \\
\\
\times T_{\chi}\left(x_{k+1}, \ldots, x_{l}\right)\left|\hat{f}_{l+1}, \ldots, \hat{f}_{m}^{\text {in }}\right\rangle \mid<\infty .
\end{aligned}
$$

Let us first outline the idea leading to (3.16). The time-ordering in $T_{\chi}\left(x_{k+1}, \ldots, x_{l}\right)$ guarantees in (3.15) that the group of field operators integrated over the $\dot{f}_{i}^{(*)}\left(x_{i}, t_{i}\right)$ with largest $t_{i}$ operates on the left on $\left|\hat{f}_{1}, \ldots, \hat{f}_{k}^{\text {out }}\right\rangle$, and those with the smallest $t_{i}$ on the right on $\left|\hat{f}_{l+1}, \ldots, \hat{f}_{m}^{\text {in }}\right\rangle$. By using non-overlapping $f_{i}$ one ensures that for almost equal $t_{i}$ the $\dot{A}^{(*)}\left(f_{i}, t_{i}\right)$ commute up to an error of o $\left(\left|t_{i}\right|^{-N}\right), N$ arbitrary, since their test functions have centers separating in space-like directions linearly in $t_{i}$. This removes the time-ordering within these groups and (3.16) follows from Theorem 2.1.

Uniform majorizations for $f_{i}(x, t)$ in $x, t$ follow from the work of RUELLE [5] and ARAKI [7] on the smooth solutions of the Klein-Gordon equation. One obtains:

$$
\begin{gathered}
\left|f_{i}(x, t)\right|<C_{M N}\left(1+\left(x_{0}-t\right)^{2}\right)^{-M / 2}\left(1+\left(\mathrm{x}^{2}+t^{2}\right)\right)^{-N / 2} \\
\text { for } \quad \mathrm{x} \notin C_{\eta}\left(f_{i}, t\right), \\
\left|f_{i}(x, t)\right|<C_{M}\left(1+\left(x_{0}-t\right)^{2}\right)^{-M / 2}\left(1+\left(\mathrm{x}^{2}+t^{2}\right)\right)^{-3 / 4} \\
\text { for } \quad \mathrm{x} \in C_{\eta}\left(f_{i}, t\right) .
\end{gathered}
$$

Here $C_{\eta}\left(f_{i}, t\right)$ is defined as the set of points $\mathbf{x}=\mathbf{p} t \omega^{-1}$, for which at least one $\left(p^{0}, \mathbf{p}\right)$ is contained in the $\eta$-neighborhood of $\operatorname{supp} \tilde{f}_{i}, \eta>0$ fixed. The constants $C_{M N}, C_{M}$ depend on $\eta$, but not on $x, t$. 
One sees that $f_{i}(x, t)$ together with all its derivatives is, for fixed $t$, strongly decreasing with the distance from its "essential support"

$$
S_{\eta}\left(f_{i}, t\right)=\left\{x: x_{0}=t, \mathbf{x} \in C_{\eta}\left(f_{i}, t\right)\right\} .
$$

For non-overlapping $f_{k+1}, \ldots, f_{l}$ all $S_{\eta}\left(f_{i}, 1 \pm \eta\right)$ are pairwise space-like separated for sufficiently small $\eta>0$. Then the $S_{\eta}\left(f_{i},\left(1 \pm \eta_{i}\right) t\right)$ move pair-wise apart in space-like direction, linearly in $t$ for $\left|\eta_{i}\right| \leqq \eta$. Using this we can majorize (3.15).

Due to the symmetry of $T_{\chi}\left(x_{k+1}, \ldots, x_{l}\right)$ we can restrict ourselves to the sector

$$
t_{k+1} \geqq t_{k+2} \geqq \cdots t_{l} .
$$

Without restriction let $t \equiv t_{k+1} \geqq\left|t_{l}\right|$. We consider a subsector fulfilling in addition

$t_{i}-t_{i+1} \leqq \frac{\eta}{4(l-k)} t \quad(k+1 \leqq i \leqq n-1), \quad t_{n}-t_{n+1}>\frac{\eta}{4(l-k)} t$

with $k+1 \leqq n \leqq l$. We claim that for all $N \geqq 0$ and all $t_{k+1}, \ldots, t_{l}$ satisfying (3.19), (3.20) the distribution in (3.15) can be replaced by $\left\langle\hat{f}_{1}, \ldots, \hat{f}_{k}^{\text {out }}\left|A\left(x_{k+1}\right), \ldots, A\left(x_{n}\right) T_{\gamma}\left(x_{n+1}, \ldots, x_{l}\right)\right| \hat{f}_{l+1}, \ldots, \hat{f}_{m}^{\text {in }}\right\rangle$

up to an error of $\mathrm{o}\left(|t|^{-N}\right)$.

For, consider a permutation $P \in \mathbb{\Xi}^{l-k}$ with $\{P(k+1), \ldots, P(n)\} \neq$ $\neq\{k+1, \ldots, n\}$. The term containing $A\left(x_{P(k+1)}\right), \ldots, A\left(x_{P(\imath)}\right)$ in (3.2) has support in $G_{P}=\left\{x_{P(i)}^{0}-x_{P(i+1)}^{0}>-R, k+1 \leqq i \leqq l-1\right\}$ for some $R=R(\chi)<\infty$. Since the time-ordering induced by $P$ is incompatible with (3.19), (3.20), one can deduce from (3.17) uniformly for all $(t) \epsilon$ $\in\{(3.19),(3.20)\}$ and $(x) \in G_{P}$ the estimate

$$
\left|P_{x} D_{x} \prod_{i=k+1}^{l} \dot{f}_{i}^{(*)}\left(x_{i}, t_{i}\right)\right|<C(1+|t|)^{-N}
$$

with $C=C\left(N, P_{x}, D_{x}\right)<\infty$ for all $N$ and all polynomials $P_{x}, D_{x}$ in the $x_{i}^{j}, \partial / \partial x_{i}^{j}$. Up to an error of o $\left(|t|^{-N}\right)$ those terms can therefore be omitted in $\{(3.19),(3.20)\}$.

Consider now a contribution to $T_{\chi}\left(x_{k+1}, \ldots, x_{l}\right)$ corresponding to a $P \in \mathfrak{S}^{l-k}$ with $\{P(k+1), \ldots, P(n)\}=\{k+1, \ldots, n\}$. Here one can replace in $(3.15)\left\langle\hat{f}_{i}, \ldots, \hat{f}_{k}^{\text {out }}\left|A\left(x_{P(k+1)}\right), \ldots, A\left(x_{P(l)}\right)\right| \hat{f}_{l+1}, \ldots, f_{m}^{\text {in }}\right\rangle$ by $\left\langle\hat{f}_{1}, \ldots, \hat{f}_{k}^{\text {out }}\left|A\left(x_{k+1}\right), \ldots, A\left(x_{n}\right) A\left(x_{P(n+1)}\right), \ldots, A\left(x_{P(l)}\right)\right| \hat{f}_{l+1}, \ldots, \hat{f}_{m}^{\text {in }}\right\rangle$. This introduces in the support of $\bigotimes_{i=k+1}^{l} f_{i}^{(*)}\left(x_{i}, t_{i}\right)$ for $(t) \in\{(3.19),(3.20)\}$ only an error of $o\left(|t|^{-N}\right)$, since $\eta>0$ was chosen sufficiently small, so that the essential supports $S_{\eta}\left(f_{i}, t_{i}\right), k+1 \leqq i \leqq n$, separate by a space-like interval increasing linearly in $|t|$. Furthermore up to o $\left(|t|^{-N}\right)$ $\theta\left(x_{P(n)}^{0}-s_{P(n)}-x_{P(n+1)}^{0}+s_{P(n+1)}\right)$ can be replaced by 1 in the timeordering (3.20). By adding the regularized $\theta$-functions up to 1 , one obtains (3.21). 
By the Schwarz inequality:

$$
\begin{aligned}
& \mid \int \prod_{i=k+1}^{l} d x_{i} \dot{f}_{i}^{(*)}\left(x_{i}, t_{i}\right)\left\langle\hat{f}_{1}, \ldots, \hat{f}_{k}^{\text {out }}\right| A\left(x_{k+1}\right), \ldots, A\left(x_{n}\right) T_{z}\left(x_{n+1}, \ldots, x_{l}\right) \times \\
& \left.\times\left|\hat{f}_{l+1}, \ldots, \hat{f}_{m}^{\text {in }}\right\rangle\left|\leqq \| \int \prod_{i=n}^{k+1} d x_{i} \hat{f}_{i}^{(*)}\left(x_{i}, t_{i}\right)^{*} A\left(x_{i}\right)\right| \hat{f}_{1}, \ldots, \hat{f}_{k}^{\text {out }}\right) \| \times \quad(3.23) \\
& \left.\times \| \int \prod_{i=n+1}^{l} d x_{i} \dot{f}_{i}^{(*)}\left(x_{i}, t_{i}\right) T_{\chi}\left(x_{n+1}, \ldots, x_{l}\right) \mid f_{l+1}, \ldots, f_{m}^{\text {in }}\right) \| .
\end{aligned}
$$

Due to the LSZ asymptotic condition the first factor is decreasing faster than any power of $|t|^{-1}$ in the subsector $\{(3.19),(3.20)\}$ for $\eta>0$ sufficiently small. Since the second factor is tempered in $t$, we obtain (3.16).

These majorizations extend to

$$
\begin{gathered}
\left\langle\mathbf{p}_{1}, \ldots, \mathbf{p}_{k}^{\text {out }}\left|\widetilde{T}_{z}\left( \pm p_{k+1}, \ldots, \pm p_{l}\right)\right| \mathbf{p}_{l+1}, \ldots, \mathbf{p}_{m}^{\text {in }}\right\rangle \times \\
\times \prod_{i=k+1}^{l}\left(p_{i}^{2}-m^{2}\right) \exp \left[i\left(p_{i}^{0}-\omega_{i}\right) t_{i}\right]
\end{gathered}
$$

integrated over test functions from $\mathscr{S}\left(R^{3(n+k-l)} \times G^{(l-k)}\right)$ with pairwise disjoint supports in the velocities $\mathbf{p}_{i} \omega_{i}^{-1}$. In the variables $p_{i}^{0}-\omega_{i}$, $\mathbf{p}_{i},(3.15)$ is equivalent to the fact that (3.14) integrated over non-overlapping $\psi \in \mathscr{D}\left(R^{3 m}\right)$ is $C^{\infty}$ in the variables $p_{i}-\omega_{i}$ in a neighborhood of the origin (depending on $\psi$ ), and at the origin for non-overlapping $\psi \in \mathscr{S}\left(R^{3 m}\right)$. This proves Theorem 3.1.

Since (3.15) is uniformly fast decreasing in $t_{k+1}, \ldots, t_{l}$, it can be integrated in any order over all $t_{k+1}, \ldots, t_{l}$. The repeated integral can be evaluated using the reduction formulae (3.4) and (3.9). This gives for non-overlapping $\left\{\tilde{f}_{i}\right\} \subset \mathscr{S}(G)$ the well-known [2] connection with the $S$-matrix :

$$
\begin{aligned}
& \left\langle\hat{f}_{1}, \ldots, \hat{f}_{n}^{\text {out }} \mid \hat{f}_{n+1}, \ldots, \hat{f}_{m}^{\text {in }}\right\rangle=(-i \sqrt{2 \pi})^{l-k} \int \prod_{i=k+1}^{l} d p_{i} \delta_{+}\left(p_{i}\right) \times \\
& \times \prod_{i=k+1}^{n} f_{i}^{*}\left(p_{i}\right) \prod_{i=n+1}^{l} \tilde{f}_{i}\left(p_{i}\right)\left[\prod_{i=k+1}^{l}\left(p_{i}^{2}-m^{2}\right) \times\right. \\
& \times\left\langle\hat{f}_{1}, \ldots, \hat{f}_{k}^{\text {out }}\left|\widetilde{T}_{\chi}\left(p_{k+1}, \ldots, p_{n},-p_{n+1}, \ldots,-p_{l}\right)\right| \hat{f}_{l+1}, \ldots, \hat{f}_{m}^{\text {in }}\right\rangle .
\end{aligned}
$$

We collect these results in the

Theorem 3.2: For $p_{i} \neq p_{j}, i \neq j$, the scattering amplitudes are related to time-ordered distributions by

$$
\begin{aligned}
& \left\langle\mathbf{p}_{1}, \ldots, \mathbf{p}_{m}^{\text {out }} \mid \mathbf{p}_{m+1}, \ldots, \mathbf{p}_{n}^{\text {in }}\right\rangle \\
& =\left.(-i \sqrt{2 \pi})^{n}\left[\prod_{i=1}^{n}\left(p_{i}^{2}-m^{2}\right)\left\langle\widetilde{T}_{\chi}\left(p_{i}, \ldots, p_{m},-p_{m+1}, \ldots,-p_{n}\right)\right\rangle_{0}\right]\right|_{p_{i}^{0}=\omega_{i}} .
\end{aligned}
$$

The off-shell extrapolations $\left[\prod_{i=1}^{n}\left(p_{i}^{2}-m^{2}\right)\left\langle\widetilde{T}_{\chi}\left(p_{1}, \ldots, p_{m},-p_{m+1}, \ldots\right.\right.\right.$, $\left.\left.\left.-p_{n}\right)\right\rangle_{0}\right]$ (depending on the regularization $\chi$ ) are $C^{\infty}$ around the energy shell, 
when integrated over $\mathbf{p}_{1}, \ldots, \mathbf{p}_{n}$ for $\mathbf{p}_{i} \omega_{i}^{-1} \neq \mathbf{p}_{j} \omega_{j}^{-1}(i \neq j)$. If sharp $i L^{+}$invariant tempered time-ordered distributions $\left\langle T_{0}\left(x_{1}, \ldots, x_{n}\right)\right\rangle_{0}$ can be defined satisfying

$$
\left.\left.\left\langle T_{0}\left(x_{1}, \ldots, x_{n}\right)\right\rangle_{0}=\left\langle A\left(x_{P(1)}\right), \ldots, A\left(x_{P(n)}\right)\right\rangle_{0} \text { for } x_{P(1)}^{0}\right\rangle \cdots\right\rangle x_{P(n)}^{0},
$$

then (3.25) remains valid in the same sense.

Remark: The last statement follows from an analysis of the proof of Theorem 3.1. As expected, possible ambiguities in the definition of $\left\langle T_{0}\left(x_{1}, \ldots, x_{n}\right)\right\rangle_{0}$ do not contribute to the energy shell relation (3.25).

In the proof of Theorem 3.1 it became apparent that for non-overlapping $\tilde{\psi} \in \mathscr{S}\left(G^{n}\right)$

$$
\begin{aligned}
F(t) & =\int \prod_{i=1}^{n} d p_{i} \exp \left[i\left(p_{i}^{0}-\omega_{i}\right) t_{i}\right] \tilde{\psi}(p) \times \\
& \times\left\langle\widetilde{T}_{\chi}\left(p_{1}, \ldots, p_{m},-p_{m+1}, \ldots,-p_{n}\right)\right\rangle_{0}
\end{aligned}
$$

converges rapidly whenever some of the $t_{1}, \ldots, t_{n}$ tend independently to $\pm \infty$. This asymptotic behavior of (3.27) for large $\left|t_{i}\right|$ can be equivalently expressed by the singularity structure of $\left\langle\tilde{T}_{\chi}\left(p_{1}, \ldots,-p_{n}\right)\right\rangle_{0}$ around the energy shell: apart from singularities of the type $\delta\left(p_{i}^{0}-\omega_{i}\right) \pm$ $\pm \frac{i}{\pi} P\left(p_{i}^{0}-\omega_{i}\right)^{-1}$ in any subset of the variables $p_{1}^{0}-\omega_{1}, \ldots, p_{n}^{0}-\omega_{n}$ $\left\langle\tilde{T}_{\chi}\left(p_{1}, \ldots,-p_{n}\right)\right\rangle_{0}$ is $C^{\infty}$ around the origin, when integrated over nonoverlapping $\tilde{\varphi} \in \mathscr{S}\left(R^{3 n}\right)$. This sheds some light on the results of GREENBERG [17] and WIGHTMAN, who derived the LSZ asymptotic condition from similar assumptions on the singularity structure of the VEV $\left\langle A\left( \pm p_{1}\right), \ldots, A\left( \pm p_{n}\right)\right\rangle_{0}$ around $p_{i}^{0}=\omega_{i}$.

$F(t)$ in (3.27) tends rapidly to zero for any $t_{i} \rightarrow-\infty$. Let $\Delta \equiv\langle-\infty, 0]^{n}$. Then $F(0)$ can be computed by the uniformly convergent integral:

$$
\begin{gathered}
F(0)=\int_{\Delta}\left[\prod_{i=1}^{n} d t_{i} \frac{\partial}{\partial t_{i}}\right] F\left(t_{1}, \ldots, t_{n}\right)=\lim _{\varepsilon_{1}, \ldots, \varepsilon_{n} \downarrow 0} \times \\
\times \int \prod_{i=1}^{n} d p_{i}\left(p_{i}^{2}-m^{2}+i \varepsilon_{i}\right)^{-1} \tilde{\psi}(p)\left[\prod_{i=1}^{n}\left(p_{i}^{2}-m^{2}\right)\left\langle\tilde{T}_{\chi}\left(p_{1}, \ldots,-p_{n}\right)\right\rangle_{0}\right] .
\end{gathered}
$$

Since there is no division problem away from the mass shell, this gives as a distribution identity the well-known formula [13].

$$
\begin{gathered}
\left\langle\widetilde{T}_{\chi}\left(p_{1}, \ldots, p_{n}\right)\right\rangle_{0}=\lim _{\varepsilon_{1}, \ldots, \varepsilon_{n} \downarrow 0} \prod_{i=1}^{n}\left(p_{i}^{2}-m^{2}+i \varepsilon_{i}\right)^{-1} \times \\
\times\left[\prod_{i=1}^{n}\left(p_{i}^{2}-m^{2}\right)\left\langle\widetilde{T}_{\chi}\left(p_{1}, \ldots, p_{n}\right)\right\rangle_{0}\right] .
\end{gathered}
$$

Theorem 3.2 and formula $(3.29)$ for $\left\langle\widetilde{T}_{\chi}\left(p_{1}, \ldots, p_{m},-p_{m+1}, \ldots,-p_{n}\right)\right\rangle_{0}$ can be immediately generalized [see $(3.11),(3.12)$ ] to the 1-particle 
structure in the variables $q_{i}=\sum_{X_{i}} p_{k}$ around the energy shell, for any partition $X_{1}, \ldots, X_{l}$ of $\{1, \ldots, n\}$.

Physically important are $2 \rightarrow n$ particle reactions. In this case one can show, as in LSZ, that the scattering amplitude can be extrapolated from the energy shell by advanced distributions $\prod_{i=1}^{n+2}\left(p_{i}^{2}-m^{2}\right)\left\langle\widetilde{A}_{\chi}\left(-p_{1}\right.\right.$; $\left.\left.-p_{2}, p_{3}, \ldots, p_{n+2}\right)\right\rangle_{0}$ for non-overlapping velocities:

Theorem 3.3: For $\mathbf{p}_{i} \omega_{i}^{-1} \neq \mathbf{p}_{j} \omega_{j}^{-1}, i \neq j$, one has the distribution identity:

$$
\begin{gathered}
\left\langle\mathbf{p}_{3}, \ldots, \mathbf{p}_{n}^{\text {out }} \mid \mathbf{p}_{1}, \mathbf{p}_{2}^{\text {in }}\right\rangle \\
=-\left.i(2 \pi)^{n / 2}\left[\prod_{i=1}^{n}\left(p_{i}^{2}-m^{2}\right)\left\langle\widetilde{A}_{\chi}\left(-p_{1} ;-p_{2}, p_{3}, \ldots, p_{n}\right)\right\rangle_{0}\right]\right|_{p_{i}^{0}=\omega_{i}}
\end{gathered}
$$

The off-shell extrapolation $\left[\prod_{i=1}^{n}\left(p_{i}^{2}-m^{2}\right)\left\langle\widetilde{A}_{\chi}\left(-p_{1} ;-p_{2}, \ldots, p_{n}\right)\right\rangle_{0}\right]$ is $C^{\infty}$ in $p_{i}^{0}-\omega_{i}$ around the origin, when integrated over $\mathbf{p}_{1}, \ldots, \mathbf{p}_{n}$ with nonoverlapping $\psi \in \mathscr{S}\left(R^{3 n}\right)$.

The proof of Theorem 3.3 follows using the same argument as in Theorem 3.1. Spectrum conditions for the $2 \rightarrow n$ reactions eliminate those terms in the development of $\left\langle A_{\chi}\left(x_{1} ; \ldots, x_{n}\right)\right\rangle_{0}$ into $\mathrm{VEV}$, for which no LSZ asymptotic condition has been proved, i. e. terms of the structure $A^{(*)}\left(f_{i}, t\right) \Phi, \Phi \in \mathfrak{P} \Omega$, which need not necessarily converge for $t \rightarrow \pm \infty$. Similar spectrum conditions are not valid in general. Therefore no further information about the singularity structure of $\left\langle\widetilde{A}_{\chi}\left( \pm p_{1}, \ldots, \pm p_{n}\right)\right\rangle_{0}$, $\left\langle\widetilde{R}_{\chi}\left( \pm p_{1}, \ldots, \pm p_{n}\right)\right\rangle_{0}$ around $p_{i}^{0}=\omega_{i}$ has been derived.

\section{Conclusion}

The results obtained in this paper are an intermediate step in understanding the asymptotic structure of quantum field theory. The tools provided by the Haag-Ruelle collision theory allow a partial justification of the LSZ framework from more fundamental physical assumptions, sufficient e. g. for the derivation of dispersion relations [18]. The asymptotic condition as an $e^{i\left(p_{0}-\omega\right) t}$ limiting procedure gives in a theory of massive particles a meaning to the retarded prescription in the YangFeldman equations and to the restriction of the amputated Green's functions to the mass shell. Difficulties connected with the LSZ asymptotic condition in canonical quantized quantum electrodynamics $[19,20]$ do not appear in our case.

It remains an open question, whether the LSZ asymptotic condition can be proved for overlapping scattering states, e. g. for $\left.\mid \hat{f}_{1}, \ldots, \hat{f}_{n}^{\mathrm{ex}}\right)$ with $\left\{\hat{f}_{i}\right\} \subset \mathscr{S}\left(R^{3}\right)$, under the assumptions of the Haag-Ruelle collision theory. In a framework of bounded local observables ARAKI [8] proved the LSZ 
asymptotic condition on all states of bounded energy. Closely connected is the question of how to interpret the reduction formulae for overlapping momenta. For separately non-overlapping incoming and outgoing momenta (3.25) remains valid in the sense of a repeated restriction to the mass shell, independent of the order of the limits. Thus the matrix elements of the unitary $S$-matrix (assuming asymptotic completeness) between a dense set of states are related to $\Pi\left(p_{i}^{2}-m^{2}\right)\left\langle\widetilde{T}_{\chi}\left(p_{1}, \ldots, p_{n}\right)\right\rangle_{0}$. This complete determination of $S$ might be sufficient for practical purposes, where the singular configuration of particles running asymptotically parallel with equal velocities are always treated as a limit. Yet in many operations with the Green's distributions, as in the use of the unitarity equations for the many-particle structure analysis [16], one needs stronger tools for handling mathematically ill-defined expressions.

The author gratefully acknowledges many stimulating discussions with Professors R. Jost and A. S. Wightman. It is a pleasure to thank Professor R. OppenHEIMER for the warm hospitality at the Institute for Advanced Study.

\section{References}

[1] Jost, R.: Proceedings of the Sienna International Conference on Elementary Particles. Bologna 1963.

[2] Lehmann, H., K. Symanzik u. W. Zimmermann: Nuovo cimento 1, 205 (1955); 6, 319 (1957); Glaser, V., H. Lehmann u. W. ZimmermanN: Nuovo cimento 6, 1122 (1957).

[3] Wightman, A. S.: Phys. Rev. 101, 860 (1957); see also: R. F. Streater and A. S. Wrghtman: PCT, Spin and Statistics, and All That. New York: Benjamin 1964; R. Jost: General Theory of Quantized Fields, Providence. Am. Math. Soc. Publications (1965).

[4] HAAG, R.: Phys. Rev. 112, 669 (1958).

[5] Ruelle, D.: Helv. Phys. Acta 35, 147 (1962).

[6] Yang, C. N., and D. Feldman: Phys. Rev. 79, 972 (1950).

[7] ARAKI, H.: Vorlesungen über axiomatische Quantenfeldtheorie, ETH. Zürich $1961 / 62$.

[8] Araki, H., K. Hepp u. D. Ruelle: Helv. Phys. Acta 35, 164 (1962).

[9] HaAg, R., u. D. W. Robinson: Private communication.

[10] Fierz, M.: Helv. Phys. Acta 12, 3 (1939).

[11] Steinmann, O.: Helv. Phys. Acta 36, 90 (1963).

[12] Schwartz, L.: Théorie des distributions, I, II. Paris 1957/59.

[13] Zimmermann, W.: Nuovo cimento 13, 503 (1959); 16, 690 (1960).

[14] RUelle, D.: Unpublished.

[15] Streater, R. F.: Nuovo cimento 25, 274 (1962).

[16] Symanzik, K.: J. Math. Physics 1, 249 (1960).

[17] Greenberg, O. W.: Thesis, Princeton 1956.

[18] Hepp, K.: Helv. Phys. Acta 37, 639 (1964).

[19] Källé́n, G.: Handbuch der Physik V, 1. Berlin, Göttingen, Heidelberg: Springer 1958.

[20] Rollnik, H., B. Stech u. E. Nunnemann: Z. Physik 159, 482 (1960).

(Received January 20, 1965) 